\title{
El análisis químico de las aguas. Ciencia colonial, exploración y supervivencia en península Valdés a fines del siglo XVIII
}

The chemical analysis of the waters. Colonial science, exploration and survival in Valdés Peninsula at the end of the 18th century

\section{Marcia Bianchi Villelli}

\section{(2) OpenEdition}

\section{Journals}

\section{Electronic version}

URL: http://journals.openedition.org/corpusarchivos/2663

DOI: $10.4000 /$ corpusarchivos.2663

ISSN: $1853-8037$

\section{Publisher}

Diego Escolar

\section{Electronic reference}

Marcia Bianchi Villelli, «El análisis químico de las aguas. Ciencia colonial, exploración y supervivencia en península Valdés a fines del siglo XVIII », Corpus [En línea], Vol. 8, No 2 | 2018, Publicado el 05 febrero 2019, consultado el 30 abril 2019. URL : http://journals.openedition.org/corpusarchivos/2663 ; DOI : 10.4000/corpusarchivos.2663

This text was automatically generated on 30 April 2019. 


\section{El análisis químico de las aguas. Ciencia colonial, exploración y} supervivencia en península Valdés a fines del siglo XVIIIMarcia Bianchi Villelli

The chemical analysis of the waters. Colonial science, exploration and survival in Valdés Peninsula at the end of the 18th century

\section{EDITOR'S NOTE}

Fecha de recepción del original: 17/5/2018

Fecha de aceptación para publicación: 12/9/2018

\section{Afianzando la presencia española en la costa patagónica}

1 Hacia 1778 y como parte de la reorganización administrativa y política de las colonias españolas, se inició el plan de poblamiento de la costa patagónica. Para esto, se definieron dos asentamientos principales, "Fuerte y Población de Nuestra Señora del Carmen" provincia de Buenos Aires-y la Nueva Población y Fuerte de Floridablanca -bahía de San Julián, provincia de Santa Cruz-, y un asentamiento subsidiario, el Fuerte San José península Valdés, provincia de Chubut. ${ }^{1}$ Se organizó el traslado de familias labradoras desde España con el fin de establecerlas en varios puntos de la costa y así afianzar la presencia de la Corona (Gorla 1984; Luiz 2006; Nacuzzi 2007; Senatore 2007; Casanueva 2013). 
2 En este trabajo nos centraremos en la trascripción y estudio de una pieza documental específica, el análisis de agua de península Valdés realizado en 1779. En primer lugar, nos introducimos en el primer año de funcionamiento del Fuerte San José y las causas alrededor del análisis de aguas, para luego comentar el contexto en que se desarrollaron. En segundo lugar, continuamos la trascripción y discutimos aspectos varios mencionados en la misma, relativos a la química y salud del siglo XVIII y, en particular, el escorbuto que fue la causa principal de muerte en los primeros meses del funcionamiento del Fuerte San José.

\section{Los asentamientos de península Valdés}

3 En enero de 1779 llegó la primera expedición con Juan de la Piedra ${ }^{2}$, Superintendente de la Costa Patagónica, con instrucciones para el relevamiento de tierras y ríos, calidad de fondeaderos y profundidades y las circunstancias del puerto. Se sumaron los hermanos Francisco y Antonio Viedma y el piloto, Basilio Villarino. Eligieron un primer campamento en el golfo San José, mientras hicieron expediciones al interior de la península Valdés en busca de agua dulce.

Por la calidad del embarcadero y la cercanía a dos salinas con agua dulce, se instalaron definitivamente en la actual Playa Fracasso (MAPA 1). En función de localizar la mejor fuente de agua en la Salina Grande, se hizo un segundo asentamiento sobre la misma, Puesto de la Fuente, situado a unos $30 \mathrm{~km}$ del primero. Durante sus 31 años de funcionamiento, se desarrolló como un puesto subsidiario de Nuestro Señora del Carmen, desde el cual se fue dando apoyo y resguardo a la creciente actividad pesquera y a la extracción de sal (Bianchi Villelli 2017). En este período, la población de la península fue variando en número, y estaba compuesta por personal militar, funcionarios, capellanes, peones y presidiarios. Aunque se levantaron algunas construcciones, el Fuerte San José y el Puesto de la Fuente se caracterizaron por la precariedad y la falta de abastecimiento (Bianchi Villelli et al. 2013, 2018; Buscaglia et al. 2016). En 1810 fueron atacados y destruidos ambos establecimientos por malones indígenas (Buscaglia et al. 2012; Buscaglia 


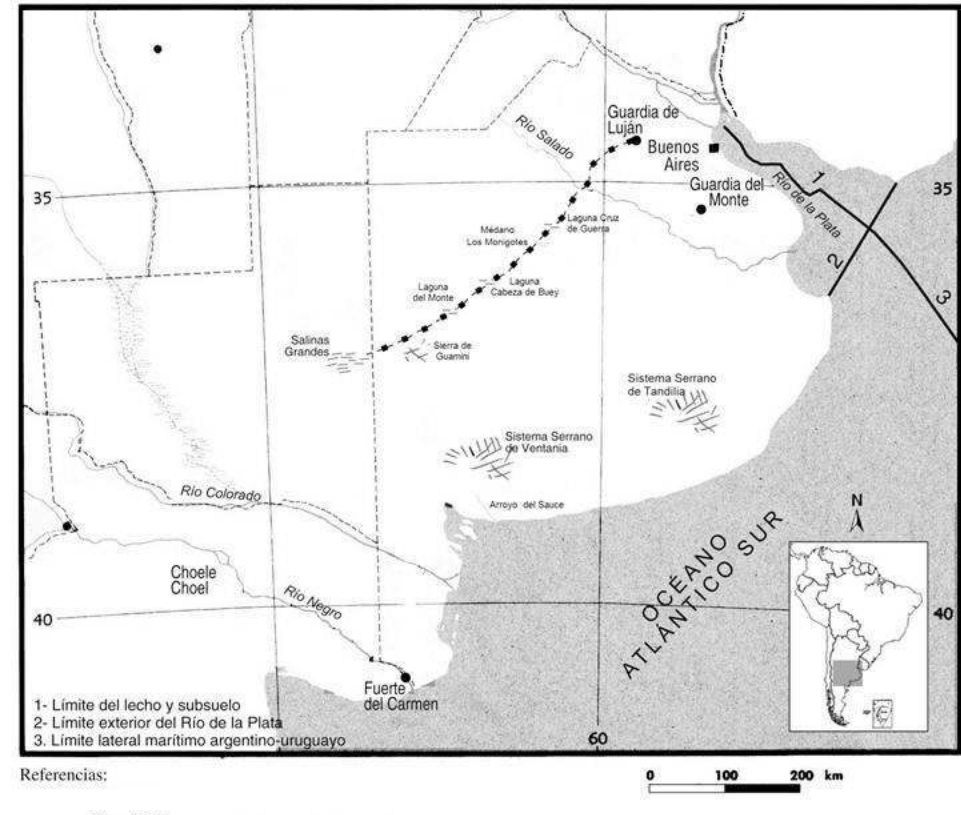

Zizur $1786 \ldots \ldots \ldots$

MAPA 1. Localización de los asentamientos coloniales de Fuerte San José (Playa Fracasso) y Puesto de la Fuente (Salina Grande), península Valdés - ambos han sido corroborados por estudios arqueológicos (Buscaglia et al. 2012; Bianchi Villelli et al. 2013).

Aquí nos centramos en el primer año de funcionamiento del Fuerte San José, el cual fue extremadamente duro, por la distancia al agua potable y por la falta de abastecimiento desde el Río de la Plata. En los meses de enero y febrero de 1779, se eligió el lugar del campamento, se realizaron los pozos en busca del agua y se avanzó con el levantamiento de las primeras habitaciones de resguardo; en esos meses Basilio Villarino escribió un Diario con su relevamiento del interior, en el que halló el manantial de agua dulce de la Salina Grande (Enrique 2015). El Cte. Juan de la Piedra decidió abandonar el campamento, ${ }^{3}$ dejando a cargo a Francisco Viedma, quien en abril se retiró a fundar el establecimiento del Río Negro con Basilio Villarino. Antonio Viedma es quien queda a cargo de sostener el asentamiento, mientras que el escorbuto comenzó a tener víctimas fatales; murieron 28 soldados como resultado del consumo de agua de los pozos cercanos al mar. ${ }^{4} \mathrm{La}$ escasez de víveres profundizó el descontento en la tropa, quienes fijaron en la puerta de la capilla un pasquín con amenazas de muerte a las dos autoridades del asentamiento, don Antonio Viedma y el capitán Nicolás García. ${ }^{5}$ En agosto, Viedma decidió levantar el asentamiento dejándolo en manos del teniente Pedro García y seis soldados, quienes optaron por permanecer allí. ${ }^{6}$

5 A su vuelta a Buenos Aires, debe informar al virrey Vértiz la causa del abandono y las circunstancias del asentamiento. ${ }^{7}$ Es en este informe que se incluyó un estudio muy particular sobre las aguas de Valdés. Durante su estadía en el Fuerte, mientras lidiaba con la alta mortalidad como resultado del escorbuto, Antonio Viedma tomó muestras de agua de los pozos que utilizaban. Una del pozo del Fuerte que tenía agua salada, y otra del pozo del manantial natural de la Salina Grande. Cuando llegó en agosto a Buenos Aires, las entregó al médico Miguel Gorman, quien las analizó y produjo el documento que aquí se presenta. El análisis de las aguas y su informe fueron un argumento importante usado por 
Viedma a la hora de dar cuenta ante el virrey Vértiz por los conflictos suscitados durante su permanencia en el asentamiento de San José.

\section{La química colonial}

6 El análisis mismo de las muestras de agua estuvo a cargo de Miguel Gorman (1749-1819), un médico irlandés llegado a Buenos Aires en 1777 como parte de la expedición del virrey Ceballos a Colonia del Sacramento; en 1779 ya era el titular del Protomedicato del Río de la Plata, rol que desempañaba cuando realiza los estudios del agua de la península (Beltrán 1937; s/a 2010; Romi 2015). El Protomedicato fue una institución colonial arraigada por una larga tradición, que buscó reorganizar y modernizar la práctica médica, botánica y de farmacia, siendo sus funciones fiscalizar el quehacer de médicos, cirujanos y boticarios (Aceves Pastrana 2004; Berra 2010; Arce 2016).

7 En el Río de la Plata, el Protomedicato se creó en 1780 con el nombramiento de Miguel Gorman como titular, quien se ocupó del saneamiento de las ciudades coloniales a partir del abastecimiento de agua potable, de la construcción de letrinas, la reglamentación de cementerios y el tratamiento de la basura; a su vez, buscó establecer los programas de formación de médicos profesionales, practicantes, sangradores, barberos y otras tareas afines. Asimismo, estableció un reglamento para inspeccionar sistemáticamente los barcos que arribaban al puerto, así como la calidad del agua que recogían los aguateros en el río. También propuso al virrey Nicolás de Arredondo practicar una variolización masiva en 1785 (Arce 2016), en virtud de que la viruela era una de las principales epidemias que afectaban a la ciudad de Buenos Aires. En 1805, implementó la vacunación obligatoria en todo el país y el aislamiento de los enfermos contagiosos (Berra 2010, p. 96).

Tanto en España como en distintas colonias -Virreinato de Nueva España, Río de la Plata, Nueva Granada- las últimas décadas del siglo XVIII llevaron a la sistematización y estandarización de la química como ciencia "moderna". ${ }^{8} \mathrm{Si}$ bien la historia de la química marca una renovación en la Ilustración, hoy en día se cuestiona firmemente este discurso "positivista", mostrando los límites que efectivamente tenía el conocimiento químico para fines del siglo XVIII (Bertomeu 2011). Algunas de las contradicciones mencionadas muestran cómo permanecía el lenguaje de la alquimia en algunos análisis ya "modernos". En particular para los análisis realizados por Gorman, es importante tener en claro que las reformas conceptuales y terminológicas que introdujo Lavoisier en su experimentación con el oxígeno como elemento químico fueron contemporáneas a este momento, por lo que no llegaron a incorporarse en el informe del médico. Por este motivo es sumamente interesante examinar los análisis específicos realizados sobre cada una de las muestras de las aguas patagónicas ya que dan cuenta de este momento transicional entre las viejas prácticas de alquimia y la química de Lavoisier.

9 Ahora bien, las muestras tomadas por Viedma corresponden a los dos asentamientos: Fuerte San José y Puesto de la Fuente -ver MAPA 1. Son dos localizaciones bien diferenciadas, la primera en Playa Fracasso, golfo San José, corresponde a uno de los pozos realizados por el personal del Fuerte para buscar agua dulce. Si bien se desconoce su profundidad y ubicación precisa, está localizado arqueológicamente en el Fuerte San José. El sitio tiene una distancia de 200 metros de la playa, lo que resulta en que la muestra de agua sea muy parecida al agua del mar. La segunda, el Puesto de la Fuente se localiza en estancia Los Manantiales, Salina Grande. Presenta varios manantiales de agua dulce, a pesar de ser tan cercano a dicha Salina. El origen de dichos manantiales fue 
estudiado desde la hidrogeología de la zona, definiendo la infiltración de las lluvias de todo el centro sur de la península, como fuente para los mismos (Álvarez et al. 2010). Como resultado, ambas muestras tienen cierto grado de salinidad, en un caso marina por la cercanía al mar, mientras que en el otro, la sal se concentraba por evaporación de lagunas naturales. ${ }^{9}$

\section{Análisis químico de las aguas} que Vértiz le solicita a Antonio Viedma cuando llega de San José. ${ }^{10}$ Se detalla a continuación del pedido formal de Vértiz del correspondiente "Análisis Chímica" de las muestras de agua traídas por Viedma desde la península, seguido del informe de Gorman. El texto está organizado con la descripción de los análisis de cada muestra en primer lugar, seguido de una discusión sobre la salinidad como base para el escorbuto, y por último, unas breves consideraciones generales sobre el impacto en la salud del consumo de aguas salobres. Está firmado por Miguel Gorman, en Buenos Aires, el 25 de agosto de 1779. Consiste en 6 folios, archivados en: AGI, Buenos Aires, Legajo 326. Ramo 2. Dictamen de Miguel Gorman. Folios 718 a 723v. Buenos Aires, 25 de agosto de 1779. El documento no está ni digitalizado ni microfilmado, fue adquirido personalmente como copia microfilmada. ${ }^{11}$

11 Aquí se presenta la transcripción total del informe. Se modernizó la ortografía y se desplegaron las abreviaturas, se indican la numeración de folios y el cambio de verso y reverso. Se ha marcado en notas al pie, la terminología moderna sobre los componentes utilizados para el análisis químico según la búsqueda bibliográfica realizada y la consulta con profesionales.

"Buenos Aires, 25 de agosto de 1779. En atención a que Don Antonio de Viedma ha manifestado con separación el agua de los pozos de la Playa, de que generalmente se surtía el establecimiento de San José, y también la de las Fuentes distantes a aquel puerto y el Don D. Miguel Gorman hará que de una y otra se ejecute el correspondiente Análisis químico, nombrando a este fin al médico y Boticario que conceptúe con la suficiente aptitud para desempeñarla; y en vista de los que éstas expusieren, me informará con individual extensión, acerca de sus cualidades, y de los efectos que puedan causar. Vertiz

Excelentísimo Señor. En cumplimiento de su superior decreto de vuestra excelencia en que me manda a, hacer los análisis de las aguas traídas de las costas patagónicas, y que

Reverso

de su resultado, exponga mi dictamen: Procedí de la forma siguiente= Reconocí primeramente las aguas próximas a la orilla del mar, valiéndome en la primera impresión de los sentidos, y aunque el agua carece por si de color, estas se manifiestan adornadas de un ligerísimo color verde: gustándolas, teñían con su impresión salobre a la boca, causando sed, después de algún tiempo.

Una libra ${ }^{12}$ medida de dicha agua, pesa más que igual cantidad de la destilada, cuatro escrúpulos, y pesa con la del río en calidad de una libra cada una, un escrúpulo más advirtiendo, que las aguas patagónicas, por el mucho tiempo que se conservaron sin movimiento en las vasijas, habrán precipitado al fondo, lo más craso de las partículas salino-férreas, que se suspendían en sus poros. Por la evaporación, resultó media onza 719 verso 
de sal en cuatro libras de agua cuya naturaleza es de sal común, o marina, según lo acreditó la figura cubica de sus sales, y la decrepitación. De estas simples inspecciones, pasé a otras más exactas, para inferir por las precipitaciones que causaron en el agua, las sustancias que se le agregaban, si contenían otro principio. Los Polvos de Agallas de Levante, ${ }^{13}$ no causaron alteración en su color, ni la tintura de violetas, ${ }^{14}$ ni la inmersión en papel azul..$^{15}$ La sal de Tártaro ${ }^{16}$ precipitó al fondo de estas aguas unas glebas blancas terrestres, ${ }^{17}$ muy análogas de la magnesia. ${ }^{18}$ Echando sobre una libra de agua, cantidad de espíritu de vitriolo, ${ }^{19}$ no existió efervescencia, e inmediatamente se precipitó al fondo en todo a la sal de Glauber, ${ }^{20}$ y volviendo a repetir, la disolución, filtración,

Reverso

evaporación y cristalización, de dicho residuo, se separó un precipitado terrestre blanco, análogo a la sal selenita. ${ }^{21}$

Echando sobre otra cantidad de agua, el espíritu de Nitro-ácido, ${ }^{22}$ que contenía disuelto en sus poros el mercurio, tiño algo de amarillo el agua y se precipitó luego al fondo formando una especie de turbit mineral. ${ }^{23}$

La solución de la plata, se precipitaba del mismo modo en la forma de un cuarzo blanco, y por la cristalización, resultó una sal metálica lunar, que llaman los químicos, Luna Cornada; ${ }^{24}$ el jabón ${ }^{25}$ que se disolvió se cortó luego en porciones gruesas, y los farináceos y verduras, salieron al cabo de seis horas de cocción, crudos, mas endurecidos, y corrugados.

Las aguas de las fuentes distan de las de la orilla como cinco a seis leguas, pesan en cantidad

720 verso

medida de una libra, con las destilada, un escrúpulo más que esta última, y menos de uno que la de río, y apenas dejaron en la evaporación algunos granos de Caput Mortum, ${ }^{26}$ vulgo asientos.

Cuecen brevemente los alimentos, disuelven el jabón, y no demuestran por el resultado de las anteriores, pruebas de contener otros principios que el salino en corta cantidad, lo que acredita más el gusto, que apenas siente la impresión, después del algún tiempo.

Resulta de las varias experiencias que se han hecho en las aguas patagónicas, que las primeras están muy cargadas de sal común, y otra calidad de sal marina a la terrestre, que con dificultad se cristaliza, por eso con la sal de tártaro, forman la magnesia, con el espíritu de vitriolo, el de Glauber, no se alteran con los ácidos ni con los alcalinos, porque no encierran en si ninguno de ellos, ni con las agallas toman

Reverso

el color negro, porque no contienen marte, ${ }^{27}$ de ninguna especie, ni menos particular sulfúreas, ni bituminosas, ni impresión de mineral, siendo la prueba más constante, las figuras cubicas de sus cristales, y la decrepitación.

Es muy natural el que sean saladas las aguas primeras, porque estando los pozos tan inmediatos a las mar, reciben de ellas las aguas que pasan por los poros de las arena, dejando a proporción que se retiran, lo salobre, como lo acreditan las segundas, que por las distancia en que se hallan son cuasi dulces, sin decidir por esto que estas últimas, tengan su origen de donde las primeras; pues se hallan fuentes saladísimas en más retirado de las mares, sin que se pueda pensar nacer de tan inmenso piélago; como también fuentes de agua dulce en medio de las islas.

Generalmente,

721 verso

son más útiles para la salud las aguas ligeras, porque molestan menos con su peso el ventrículo y estando menos cargas de partes heterogéneas, son por consiguiente más penetrantes, proporcionadas a la micción, y más propensas a enfilarse por los vasos mínimos mediante la fuerza motriz, sin que destruya mi argumento lo singular de más naturaleza, en quienes aprovecha menor el agua gruesa. Aunque se observa algunas veces que aun con las mejores aguas, resisten a la cocción los 
alimentos, y principalmente las farináceos, es preciso advertir que depende de la tenacidad de estos, o de estar muy envejecidos, pues lo efectos de las buenas aguas, son de acelerar la cocción de los manjares por que supone en ellas el dote de fácil división, movilidad y penetración, acreditando así su vitalidad, para el uso interno. Es vulgar y decidida opinión que el

Reverso

escorbuto lo produce la sal marina; pero este dictamen lo admitirán lo que no hubiesen leído con atención a Pringle, y Lind, ${ }^{28}$ cuya sólida teórica desvanece cualquier duda en el particular: los que han tratado el escorbuto en las navegaciones dilatadas, son más dispuestos a convencerse en que es la causa de tan horrible el mal uso de las sal solamente; pues fuera de que todas van continuamente rodeados de una atmósfera salobre, por las exhalaciones que del mar se levantan, y que al mismo tiempo, en lo más de los buques todo es tan salado; adviertan que este molesto achaque, raras veces, y casi nunca, molesta a los oficiales, porque pueden evitar otras causas, que son las más poderosas para nutrir esta hidra. El continuo uso de la sal en los alimentos de que usamos, aunque los miramos como indispensable para la sazón, es utilísimo a nuestra

722 verso

naturaleza, porque es un digestivo admirable, y un insigne antipútrido que se opone a la alcalescencia, mediante el ácido marino, ${ }^{29}$ con algo de vitriólico, que se esconde, no siendo en mucha cantidad ella, preserva carnes, peces, y frutos, de la corrupción, y las conservas, por dilatado tiempo, para incentivos a la gula, o socorros de la necesidad y finalmente es el verdadero bálsamo con que los egipcios preservaran de las influencias del tiempo los cadáveres de sus mayores.

Tan excelentes prerrogativas no destruyen los considerables daños, que induciría en la salud, la abundancia de esta sal, pues daría origen a una animonia muniatica, ${ }^{30}$ precipitando la sangre, suero y demás humores, a una disolución escorbútica particular, bien que para este último morbo, se deben agregar varios con causas Reverso

como llevo insinuando en abstracto.

Se infiere de lo dicho el dictamen que debo exponer a V.E. sobre el efecto de estas aguas, en los habitantes de las nuevas colonias patagónicas, pues siendo el principal, y el más necesario entre los alimentos es el objeto más digno de más consideración para poner a cubierto, tantas vidas como peligrarían, probablemente, según se infiere, sin violencia, y la experiencia tiene acreditado.

Las aguas situadas en la orilla del mar estando (como resultan de las evaporaciones) están sumamente cargadas de sal, y partículas férreas, siendo por consiguiente gruesas, muy pesadas, e impropias para cocer los alimentos, las juzgo, no solamente inútiles pero perjudiciales a los nuevos colonos

723 verso

porque darían margen a malas digestiones, y a un Chile grueso desigual y crudo, impropio a sufrir las mutaciones de la segunda cocción, y de aquí se originarían obstrucciones, fiebres, hipocondrías, extenuaciones y otros males a que contribuirían, no poco, lo sensible de las estaciones en aquel clima, la poca bondad de los alimentos, habitaciones, y demás auxilios, la falta de vegetales y las pasiones de ánimos, tan propensas en esos destinos.

Las aguas de las fuentes, y que distan de cinco a seis leguas de las primeras; son muy a propósito para el uso de las vidas, por ser mucho más ligeras, y cuasi imperceptiblemente cargadas de sal, y como esta, estando diluida en mucho vehículo, es utilísima con la corrupción, es digestiva, y obra como aperitivo, no hay duda, que se debe considerar con estimación, mayormente gozando la propiedad de cocer con facilidad los alimentos,

Reverso

los que siendo de buena calidad, los más selectos y apropiados, según lo permitan las circunstancias, y procurando tomar los recursos correspondientes, para ponerse 
a cubierto, y templar en lo posible las injurias de los tiempos, creo que en lo sucesivo no se experimentaran los estragos que hasta aquí hay en la salud.

Por no molestar la atención de Vuestra Excelencia ocupada en tan vastos asuntos, me he ceñido lo posible, en un material que da mucho margen, para las más curiosas y útiles reflexiones: aun en tan limitado tiempo, deseo sea del agrado de usted y tendré suma satisfacción de haber contribuido por mi parte al bien de la humanidad, y ventajas, en cuanto de la orden de Vuestra Excelencia."

Don Miguel Gorman

Es copia fiel

Antonio Viedma

\section{Sobre los análisis en sí y su método}

"Como el examen físico de las aguas suele manifestar alguno de sus principios y facilita además el conocimiento de su buena o mala cualidad es indispensable empezar por este examen preliminar, esto es reconocer a primera vista por los sentidos si el agua tiene algún olor color y sabor sensible y notable porque si carece de los caracteres dichos se puede asegurar que el agua es potable pero si tiene alguno de ellos y además cuece mal las legumbres debe tenerse por cierto que no es buena para el uso común. Los caracteres físicos de las aguas dan más ideas que las que algunos creen para su conocimiento porque descubren las aguas que son acídulas por el ácido carbónico y por el hidrógeno sulfurado las que tienen hierro en disolución y esto sirve de preliminar para tantearlas y confirmarlas luego por los reactivos. (Bañares 1820, p. 24)"31

13 El examen de Gorman es un análisis cualitativo comparativo. Como se aprecia en la cita anterior, con un método ya establecido para la medicina y la química, va evaluando las características de sabor y olor, el peso específico, la presencia de sólidos totales. Luego, a partir de disolución, filtración, evaporación y cristalización y del uso de reactivos, se evalúa la presencia de carbonatos, sulfatos, cloruros, acidez, dureza y por último, la capacidad de cocción de vegetales. En términos generales, se utilizan los nombres vulgares, y no llega a aparecer la nomenclatura de Linneo ni la de Lavoisier (Aceves Pastrana 2004); en 1820 todavía se debatía la incorporación de la nueva terminología química en farmacia (Bertomeu y Muñoz 2010; Bertomeu 2015, p. 223).

14 Así, Gorman define para una primera aproximación a partir de los sentidos: el color es apreciado por él mismo a simple vista, así como el gusto. La segunda etapa ya tiene un método y unidades de medida: el peso específico ya tiene una estandarización con agua destilada y agua del río, así como en las unidades de medidas -escrúpulos y libras. Y los sólidos totales se comparan en peso a partir de la evaporación, detectando cristales de sal y Caput Mortum.

15 Luego Gorman utiliza distintos reactivos muy comunes en la época (Bañares 1820; Montané Marti 1998, Armijo de Castro 2012):

1. Comienza con el polvo de Agallas de Levante - alto contenido de taninos- para detectar la presencia de marte - sulfuro ferroso.

2. La tintura de violetas y papel azul indican el estado ácido-base.

3. Busca determinar carbonatos con sal de Tártaro.

4. Los sulfuros con el vitriolo.

5. Los sulfatos con la comparación a la sal de Glauber y la selenita; así como con el espíritu de nitro-ácido.

6. Los cloruros se identifican a partir de la reacción con solución de plata, identificando la Luna Cornada-cloruro de plata. 
7. La dureza del agua es medida a partir del jabón: su deshidratación y resistencia, aunque aún no estaba estandarizada la composición de este.

8. La capacidad de cocción de vegetales en un tiempo determinado así como la evaluación del producto final - "salieron al cabo de seis horas de cocción, crudos, mas endurecidos, y corrugados" (Folio 719, reverso).

El informe se completa con la comparación entre ambas muestras, diferenciando claramente ambas fuentes: "Las aguas situadas en la orilla del mar (...) están sumamente cargadas de sal, y partículas férreas, siendo por consiguiente gruesas, muy pesadas, e impropias para cocer los alimentos, las juzgo, no solamente inútiles pero perjudiciales a los nuevos colonos porque darían margen a malas digestiones, (...) que contribuirían, no poco, lo sensible de las estaciones en aquel clima, la poca bondad de los alimentos, habitaciones, y demás auxilios, la falta de vegetales y las pasiones de ánimos, tan propensas en esos destinos" (Folio 22 reverso y 723 verso).

Así, las aguas cercanas al mar, son más saladas con una ligera tonalidad verde, y son 4.76 gramos más pesadas que el agua destilada. Los sólidos -cristales cúbicos de sal- son 0.2 gramos de sal por gramo de agua. La muestra no presentó contenido de hierro, y sí un estado ácido, base neutro. Sí se hallaron carbonatos -carbonato potásico y de magnesio -, sulfatos - sulfato de sodio, de calcio y mercurial-. De los cloruros, se detectó cloruro de plata. El agua resultó dura y mala para la cocción. Las aguas de los manantiales tienen un gusto dulce y pesan solo 1.19 gramos más que el agua destilada. Se aprecia una diferencia en la sal terrestre -que se cristaliza con dificultad- y como la anterior, no presenta restos ferrosos, es levemente alcalina y se hallaron los mismos carbonatos, sulfuros, sulfatos y cloruros que en la anterior. ${ }^{32}$

Es interesante observar cómo en su evaluación de las aguas, Gorman incluye las condiciones del clima, el estado de los alimentos y las condiciones precarias de habitación. El agua de las fuentes, ligera, casi sin sal, sumado a una mejor provisión de alimentos frescos y habitaciones resultarían - dice Gorman- en que no se sigan experimentado los severos problemas de salud acontecidos durante ese año en San José. Profundiza esta diferencia con las consecuencias del consumo de sal en la salud: "molestan menos con su peso el ventrículo y estando menos cargas de partes heterogéneas, son por consiguiente más penetrantes, proporcionadas a la micción, y más propensas a enfilarse por los vasos mínimos mediante la fuerza motriz" (folio 721). No obstante, reconoce la importancia de la sal para la salud y la cocción de vegetales, siempre que sea consumida sin excesos.

Luego sigue la argumentación más novedosa en relación con el escorbuto. Vale aclarar que el escorbuto es un trastorno nutricional secundario al déficit corporal de vitamina $C$ -ácido ascórbico. A fines del siglo XVIII, fue una de las complicaciones más serias que afrontaban las Coronas en sus traslados a las colonias. Recién a fines del siglo XVIII las experimentaciones dieron resultados parciales positivos, principalmente en Inglaterra.

James Lind, fue el médico inglés que en 1753 logró relacionar el escorbuto con la falta de productos frescos, fundamentalmente los cítricos. No obstante, no llegó a superar las dificultades para conservar la vitamina $\mathrm{C}$ a lo largo del tiempo. El capitán Cook fue uno de los primeros en seguir sus indicaciones y obligó a las tripulaciones a comer cítricos, evitando la muerte de sus marineros por escorbuto. De ahí en más la armada inglesa fue incorporando los cítricos en su dieta de altamar, logrando reducir drásticamente la mortandad por escorbuto. 
John Pringle, médico escoces, fue quien destacó estos avances científicos en 1776, en su discurso en la Royal Society (Torres Santo Domingo 2003). No obstante, recién en 1795 se pudo comprobar la relación entre escorbuto y consumo de vitamina C, cuando Gilbert Blane, médico dedicado a la salud de la Armada Naval inglesa, logró preservar las cualidades del jugo de cítricos en alcohol destilado (Rizzi 2010).

Por su parte, Gorman cita directamente a Lind y Pringle, mostrando con cierta obviedad que debían ser conocidos. Su punto de vista discutía con los remanentes de la teoría de humores, ${ }^{33}$ descartando que la atmósfera marina sea lo que produce el escorbuto, “(...) van continuamente rodeados de una atmósfera salobre, por las exhalaciones que del mar se levantan (...)" -folio 721 reverso. Aún más, extiende su argumento “(...) este molesto achaque, raras veces, y casi nunca, molesta a los oficiales, porque pueden evitar otras causas, que son las más poderosas para nutrir esta hidra" -folio 722 verso. Es decir, que este mal que aquejaba a las tripulaciones de todas las potencias europeas podría deberse a deficiencias en la alimentación resultado de las diferencias sociales al interior de la tripulación. En el caso de San José, los muertos por escorbuto en el primer año fueron 24 (García Guraieb et al. 2017).

Gorman concluyó sobre el uso de ambas fuentes de agua. Las de los manantiales, asociadas a buena alimentación y habitación, mejorarían considerablemente la salud; mientras que las del golfo, sumadas a mala alimentación y habitaciones precarias, llevarían nuevamente al deterioro de los habitantes del Fuerte. Esta conclusión es muy significativa en varias dimensiones. ${ }^{34}$

Por un lado, su diagnóstico de las aguas permite a Gorman extenderse aún más y dictaminar sobre las necesidades de mejores condiciones en contextos coloniales marginales como es el caso, reclamando buenos alimentos y mejoras en la infraestructura. Recordemos también que la solicitud de análisis de las aguas responde a la situación crítica atravesada por el establecimiento en el invierno de 1779 con varios fallecidos y un motín a la autoridad de Antonio Viedma. Es interesante tener en cuenta que, a pesar de haber tenido varios informes posteriores de evaluación de los asentamientos, este informe sobre la potabilidad de las aguas no fue mencionado ni considerado, acentuando la idea de desajuste entre el diseño de políticas coloniales y la puesta en práctica en el caso de este establecimiento patagónico (Bianchi Villelli 2017). Es decir, que las indicaciones de Gorman podrían haber sido incorporadas en los informes y discusiones que se desarrollaron en esos primeros años del plan de poblamiento patagónico.

5 Como ya mencionamos, las condiciones precarias e inestables fueron el escenario constante en los establecimientos de la costa patagónica. Las demandas han sido por el mejoramiento de las condiciones de habitación y animales para el traslado de agua de los manantiales; sin embargo, hay que mencionar que desde 1780 no hay más fallecidos por escorbuto en San José (García Guraieb et al. 2017).

Aún más, esta situación de precariedad no solo persistió en San José, sino que se reiteró en los asentamientos creados con posterioridad: en particular es llamativo que la alta mortalidad por escorbuto se repitió en el breve campamento en Puerto Deseado a comienzos de 1780 (Viedma 1780; Buscaglia 2012) y en el primer año de Floridablanca, 1780/1781 (Viedma 1780; Senatore 2007) -ambos bajo la autoridad de Antonio Viedma. En este sentido, llama considerablemente la atención el desfasaje entre las conclusiones 
del informe y los eventos de alta mortalidad que se reiteraron el año siguiente en los inicios de las poblaciones coloniales de la costa patagónica.

\section{Comentarios Finales}

El proyecto ilustrado es una de las trayectorias principales de la modernidad/ colonialidad; el siglo XVIII en Sudamérica se presenta como escenario de nuevas exploraciones, de hecho, con miradas que integraron la ciencia con la geopolítica (Pratt 2010). Por un lado, hubo un auge de los viajes científicos organizados desde distintas metrópolis como los de La Condamine, Humboldt, Malaspina y Félix de Azara, entre otros (Pratt 2010; Figueroa 2011; Monge 2002; Baeza y González 2004; Galera Gómez 2010). Los avances en química, medicina, biología y zoología no quedan por fuera de este escenario ya que "el proyecto de un inventario del mundo no se puede separar de la conquista y el control de buena parte del planeta por parte de las naciones más fuertes de Europa" (Nieto Olarte 2003, p. 240, Martínez 2015, p. 2). Este inventario, en el caso de Patagonia, implicó tanto que se omitan los desarrollos culturales preexistentes como que se desdibuje el funcionamiento de un discurso científico vinculado a la posesión colonial.

La colonización de península Valdés se incorporó a este inventario desde el análisis de aguas de Gorman así como las exploraciones y cartografías realizadas, no ya con una mirada científica sino con fines geopolíticos (Luiz 2006). Los mapas de Villarino, De La Piedra, Pedro García y Bernardo Tafor constituyen el corpus cartográfico para demarcación, denominación y apropiación del territorio (Bianchi Villelli 2016). La posibilidad de indagar en la puesta en práctica de los establecimientos patagónicos permite cuestionar la forma y el alcance del discurso ilustrado y moderno. En este caso, exploración, colonización, química colonial y salud se entrecruzan y muestran nuevamente los desfasajes y contradicciones entre las planificaciones y el desarrollo en la práctica de los asentamientos patagónicos

\section{Agradecimientos}

Las investigaciones fueron financiadas por los siguientes proyectos de Sistema Nacional de Ciencia y Técnica: PICT 2010-050, FONCYT 2011-1012 (directora Dra. M. Bianchi Villelli), PIP 0183 CONICET 2011-201 (dirigido por la Dra. S. Buscaglia) y PIP 20119 (dirigido por la Dra. S. Buscaglia y codirigido por la Dra. M. Bianchi Villelli). Mi agradecimiento por comentarios y colaboraciones para Silvana Buscaglia, Luciana Carignano, Solange Fernández Do Río, Laura Caruso Fermé, Bruno Sancci y Solana García Guraieb. A los evaluadores anónimos. 


\section{Fuentes}

\section{BIBLIOGRAPHY}

Aceves Pastrana, P. (2004). La Farmacia en el periodo colonial. Anales de la Real Academia Nacional de Farmacia, 70 (1), 125-145.

Achim, M. (2007). Science in translation: the commerce of facts and artifacts in the transatlantic Spanish world. Journal of Spanish Cultural Studies, 8 (2),107-115.

Alioto, S. (2011). Indios y ganado en la frontera. La ruta del río Negro (1750-1830). Rosario: Prohistoria Ediciones.

Álvarez, G. (2010). Las conexiones entre el pensamiento de Alejandro Malaspina y la representación visual de la expedición en la Patagonia (1789-1794). Magallania, 38 (1), 5-18.

Álvarez, M. del P., Trovatto, M. M., González, N. y Hernández, M. A. (2010). Comportamiento hidroquímico del agua subterránea en el Sector Sur de Península Valdés, Chubut, Argentina. I Congreso Internacional de Hidrología de Llanuras, (pp. 372-373). Azul: Ed. Martín.

Alvarez, M. del P. y Gueli, M. (2015). El rol del agua en la historia de la Península Valdés. Cuadernos de Historia Patagónica, 3, 125-136. Centro de Estudios Históricos y Sociales..

Andrade Lima, T. (1996). Humores e odores: ordem corporal e ordem social no Rio de Janeiro, século XIX. História, Ciências, Saúde-Manguinhos, 2 (3), 44-94.

Arce, H. E. (2012). A propósito del Bicentenario. Ciencias de la Salud. 6 (1), 33-34. Fundación Barceló.

Armijo de Castro, F. (2012). El análisis Químico de las aguas en el Mundo. Cien años de análisis de las aguas mineromedicinales, Balnea, 2012, 5, 213-222. ). Disponible:

https://www.ucm.es/data/cont/docs/680-2015-12-18-N\%C3\%BAmero\%205\%20(2012)\%20Cien\% 20a\%C3\%B1os\%20de\%20an\%C3\%A1lisis\%20de\%20las\%20aguas\%20mm.pdf

Balaguer Perigüell, E. (2006). La ciencia en la españa ilustrada. Canelobre, 14-35.

Bañares, G. (1820). Analisis del agua mineral de los Baños de la Fuensanta ó Hervideros, sitos en la dehesa de Villafranca propia de la encomienda de la Clavería de Calatrava en la Mancha ... en la Imprenta de D. Leonardo Núñez de Vargas. Disponible: https://books.google.com.ar/books? id=76Dr4bPJoc0C\&dq=Polvos+de+Agallas+de+Levante\&hl=es\&source=gbs_navlinks_s

Barba Ruiz, L. (2009). Quiénes colonizaron la Patagonia en el siglo XVIII. Trelew: Biblioteca Popular A. Álvarez.

Barrera, A. (2006). Empire and Knowledge: Reporting from the New World. Colonial Latin American Review, 15 (1), 39-54.

Beltrán, J. R. (1937). Historia del Protomedicato de Buenos Aires: Estado de los conocimientos sobre medicina en el Río de la Plata durante la época colonial. Buenos Aires: Editorial El Ateneo. 
Berra, H. (2010). La medicina rioplatense en 1810. Revista Médica de Rosario, 76, 94-102.

Bertomeu, J. R y Muñoz, R. (2010). Resistencias, novedades y negociaciones: la terminología química durante la primera mitad del siglo XIX en España. Dynamis, 30, 213-238.

Bertomeu, J. R. (2011). Química y esfera pública durante la Ilustración en España. En El año de la Química. Del pasado al presente. Revista Eidon, 35. Disponible: http://www.revistaeidon.es/ archivo/el-ano-de-la-quimica/del-pasado-al-presente/117775-quimica-y-esfera-publica-durantela-ilustracion-en-espana

Bertomeu, J. R. (2015). Fugaces novedades y largas persistencias: la terminología química y la profesión farmacéutica durante la primera mitad del siglo XIX. En J. Pinilla, B. Lépinette (Eds.), Traducción y difusión de la ciencia y la técnica en España (siglos XVI-XIX) (pp. 207-229). Valencia: Universitat de València.

Bianchi Villelli, M., Buscaglia, S. y Sancci, B. (2013). Una genealogía de los planos históricos de los asentamientos coloniales de Fuerte San José, Península Valdés (Siglo XVIII). Corpus. Archivos virtuales de la alteridad americana, III (1), 1-14.

Bianchi Villelli, M. (2016). Construyendo Patagonia colonial. Las cartografías de la costa patagónica (fines del siglo XVIII). En P. Nuñez (Comp.), Fronteras Conceptuales/ Fronteras Patagónicas (pp. 85-108). Bariloche: Instituto de Investigaciones en Diversidad y Procesos de Cambio. CONICET-UNRN. Río Negro.

Bianchi Villelli, M. (2017). Colonialismo en Península Valdés: entre los proyectos defensivos y las tentativas comerciales (Patagonia norte, fines del siglo XVIII). Memoria Americana, 25(1), 47-75.

Biedma, J. (1905). Crónica histórica del Río Negro. Buenos Aires: Contes.

Buscaglia, S. (2012). Poder y dinámica interétnica en la colonia española de Floridablanca. Una perspectiva histórica y arqueológica (Patagonia, Argentina, Siglo XVIII). Saarbrücken: Editorial Académica Española.

Buscaglia, S., Bianchi Villelli, M., Starópoli, L., Bosoni, C., Carelli, S. y Alberti, J. (2012). Arqueología Histórica en Península Valdés. Primeros Abordajes Históricos y Arqueológicos al Fuerte San José (1779-1810). Revista de Arqueología Histórica Argentina y Latinoamericana, 6, 47-79.

Buscaglia, S. (2015). Relaciones Interétnicas en el Fuerte San José (Patagonia, Siglo XVIII). Una Aproximación Comparativa. Revista Española de Antropología Americana, 45, 91-111.

Buscaglia, S., Alberti, J., Álvarez, M. (2016). Techno-morphological and use-wear analyses of gunflints from two Spanish colonial sites (Patagonia, Argentina). Archaeometry, 58, 230-245.

Casanueva, M. L. (2013). Colonos e Indígenas por Tierras Patagónicas. Una mirada arqueológica de la vida cotidiana transcurrida durante los siglos XVIII, XIX y XX. Saarbucken: Publicia.

Chamizo, J. A. (2004). Apuntes sobre la historia de la química en América Latina. Historia y desarrollo de la química en México. Revista Social de Química de México, 48, 165-171.

De Vos, P. (2007). From herbs to alchemy: the introduction of chemical medicine to Mexican pharmacies in the Seventeenth and Eighteenth Centuries. Journal of Spanish Cultural Studies, 8 (2), 135-168.

Enrique, L. A. (2015). Un diario de viaje inédito de Basilio Villarino y el mapa de la travesía: más de un siglo de periplo por los archivos, Corpus-Archivos virtuales de la alteridad americana, 5 (1).

Enrique, L. A. (2018). Huellas del paisaje colonial en las narrativas fundacionales sobre la frontera sur. SAA. Disponible: 
http://www.saantropologia.com.ar/wp-content/uploads/2018/08/Libro-Ayl\%C3\%A9nEnrique-2018.pdf

Entraigas, R. (1960). El fuerte del Río Negro. Buenos Aires: Librería Don Bosco.

Figueroa, M. (2011). En los márgenes del Imperio Español y de la Historia natural: Félix de Azara colector (1787-1789). Prohistoria, 15. Disponible: http://www.redalyc.org/articulo.oa? $\mathrm{id}=380135843001>$

Galera Gómez, A. (2010). Las corbetas del Rey. El viaje alrededor del mundo de Alejandro Malaspina (1789-1794). Bilbao: Fundación BBVA. http://www.fbbva.es/TLFU/tlfu/esp/publicaciones/libros/ fichalibro/index.jsp?codigo $=584$

García Guraieb, S., Tessone, A., Buscaglia, S., Crespo, C., Bianchi Villelli, M., Del Papa, M. (2016). Análisis Bioarqueológico de un Individuo Recuperado en el Fuerte San José (Pla. Valdés, Pcia. De Chubut, 1779-1810). Revista del Museo de Antropología, 61-76.

Gorla, C. (1984). Los Establecimientos Españoles en la Patagonia. Estudio Institucional. Sevilla: Escuela de Estudios Hispanoamericanos de Sevilla, Consejo Superior de Investigaciones Científicas.

Guyton de Morveau, L. B. (2009 [1788]) Lecciones de química teórica y práctica dispuestas por un nuevo método...para servir a los cursos públicos de la Academia de Dijon. Imprenta de Don Antonio Espinosa, [digitalizado por Universidad Complutense de Madrid]. Disponible:

https://books.google.com.ar/books?id=1Lk_Cq- zCa4C\&dq=la+figura+cubica+de+sus +sales\&hl=es\&source=gbs_navlinks_s]

Jourdan, A. J. L. (1829). Farmacopea universal ó Reunión comparativa de las farmacopeas de Amsterdam. Amberes: Imp. de Ramón Vergés.

Larrosa, A. (1993). Azarosos trajinar de hospicios y enfermerías en el Montevideo del Siglo XVIII. Revista Dirección Nacional de Sanidad de las Fuerzas Armadas, 16 (1), 54-61

Luiz, M. T. (2006). Relaciones fronterizas en Patagonia. La convivencia hispano-indígena a fines del período colonial Ushuaia: Universidad Nacional de la Patagonia San Juan Bosco.

Martínez, C. (2015). Usos del pasado y confiabilidad de las fuentes: Antoine-Joseph Pernety y la disputa sobre la naturaleza de América en el siglo XVIII. Corpus, 5 (2), consultado el 02 octubre 2016.

Monge, F. (2002). En la costa de la niebla. El paisaje y el discurso etnográfico ilustrado en la expedición Malaspina en el Pacífico. Madrid: Consejo Superior de Investigaciones Científicas.

Montané Martí, J.C. (1998). Diccionario para la lectura de textos coloniales en México. Sonora: Hermosillo.

Nacuzzi, L. (2007). Los grupos nómades de la Patagonia y el Chaco en el siglo XVIII: identidades, espacios, movimientos y recursos económicos ante la situación de contacto. Una reflexión comparativa. Chungara 39 (2), 221-234.

Nieto Olarte, M. (2003). Historia Natural y la Apropiación del Nuevo Mundo en la Ilustración española, Bulletin de l'Institut français d'études andines, 32 (3), Publicado el 08 diciembre 2003, consultado el 16 noviembre 2018. DOI: 10.4000/bifea.6049

Pratt, M. L. (2010). Ojos Imperiales. Buenos Aires: Fondo de Cultura Económica.

Ramos Pérez, D. (1984). El sistema de creación de establecimientos en la época de Carlos III y su carácter anti-tradicional; el caso de la costa patagónica. En Estructuras, gobierno y agentes de la administración en la América española (siglos XVI, XVII y XVIII), (pp. 503-529). Valladolid: Universidad de Valladolid. 
Rizzi, M. (2010). Historia del escorbuto. Especial referencia a las epidemias acaecidas en los sitios de Montevideo. Revista Faso 17 (2), 52-58.

Romi, J. C. (2015). Historia del Cuerpo Médico Forense de la Justicia Nacional. s/d?

Sagredo Baeza, R. y González Leiva, J. I. (2004). La expedición Malaspina en la frontera austral del imperio español. Santiago de Chile: Editorial Universitaria.

Senatore, M. X. (2007). Arqueología e Historia en la Colonia Española de Floridablanca. Patagonia, Siglo XVIII. Buenos Aires: Editorial Teseo.

Senatore, M. X., Bianchi Villelli, M., Marschoff, M., Buscaglia, S., Nuviala, V., Bosoni, C. y Staropoli, L. (2008). Una arqueología de las prácticas cotidianas en la colonia española de Floridablanca (Patagonia, Siglo XVIII). En L. Borrero, y N. Franco (Comps.), Arqueología del Extremo Sur de Sudamérica. Resultados de nuevos proyectos, (pp. 81-117). Buenos Aires: Dunken.

Sin Autor (2010). Caveat lector. Medicina (Buenos Aires), 70(1), 89-90.

Torres Santo Domingo, M. (2003). Los viajes del capitán Cook en el siglo XVIII. Una revisión bibliográfica. Biblio 3W, Revista Bibliográfica de Geografía y Ciencias Sociales, VIII (441). Disponible: http://www.ub.es/geocrit/b3w-441.htm.

Viedma, A. (1972 [1783]). Descripción de la Costa Meridional del Sur, llamada vulgarmente Patagónica (...). En Colección Pedro De Angelis, tomo viii B, (pp, 845-936). Buenos Aires: Plus Ultra.

Zusman, P. (1999). ¿Terra Australis - "Res Nullius"? El avance de la frontera colonial hispánica en la Patagonia (1778-1784). Scripta Nova, 45 (34). Disponible: http:// www.ub.es/geocrit/ sn-45-34.htm. Consultado el 30 de mayo de 2017.

Zusman, P. (2001). Entre el lugar y la línea: la constitución de las fronteras coloniales patagónicas 1780-1792. Fronteras de la Historia, 6, 41-67.

\section{NOTES}

1. Para contextualizar el plan de poblamiento español en la costa patagónica y sus diversos asentamientos, ver, entre otros, Entraigas (1960); Ramos Pérez (1984); Senatore et al.( 2008); Alioto (2011); Bianchi Villelli et al. (2013); Casanueva (2013); Bianchi Villelli (2016, 2017); Buscaglia y Bianchi Villelli (2016); Enrique (2018).

2. Archivo Histórico Nacional (en adelante AHN) Estado, legajo 2316. Diario de la expedición del mando del Comisario Superintendente Don Juan de la Piedra, Buenos Aires, 14/3/1779.

3. Fundaçao Biblioteca Nacional (en adelante FBN) MS-508 39, doc.1142. Motivos y causas para desamparar el puerto San José incluyendo documentos que manifiestan acaecimientos y diferencias suscitadas en aquel establecimiento, Montevideo, 31/8/1779.

4. Archivo General Indias (en adelante AGI), Bs As, 326. Ramo 2. Informe de Antonio Viedma sobre Puerto San José. Folios 724 a 728. Buenos Aires, 28 de septiembre de 1779.

5. Puerto San José, 23 de julio de 1779. Motivos y causas para desamparar el puerto San José..., Montevideo, 31/8/1779 (FBN MS-508 39, doc.1142). Carta 210, Buenos Aires, 8/10/1779 (AGI, Bs. As. leg. 326). Ver también Biedma (1905) y Barba Ruiz (2009).

6. Archivo general de la Nación (en adelante AGN). Sala IX, 16.3.4. Carta de P. García a F. Viedma. Puesto del Puerto de San José, 28/5/1780.

7. AGI, Buenos Aires, Leg. 326. Informe de Antonio Viedma sobre Puerto San José. Buenos Aires, 28 de septiembre de 1779 . 
8. "Los intentos de reforma de la terminología química del siglo XVIII culminaron con la obra de Louis-Bernard Guyton de Morveau, Antoine Laurent Lavoisier, Antoine Fourcroy y Claude Louis Berthollet publicada en 1787" (Bertomeu y Muñoz 2010, p. 215).

9. Es importante mencionar que el agua subterránea en la península se encuentra conformando un acuífero libre (Álvarez et al. 2010). "En los campos medanosos del sur de la península se encuentra el agua de mejor calidad. En las pequeñas acumulaciones costeras y en los manantiales de la Salina Grande el agua es dulce, pero de menor calidad. En las cercanías de la Caleta Valdés es muy salada y en el resto de la península el agua es salobre, es decir no apta para consumo humano, pero sí para la ganadería" (Álvarez y Gueli 2015, p. 132).

10. AGI, Buenos Aires, Leg. 326. Informe de Antonio Viedma sobre Puerto San José. Buenos Aires, 28 de septiembre de 1779.

11. Agradecemos al Lic. B. Sancci quién nos facilitó las copias seleccionadas por nosotras.

12. Unidades de medida de peso. Una libra equivale a $0.4536 \mathrm{kilo}=16$ onzas 96 dracmas $=5.760$ granos. Un escrúpulo: equivale a $1 / 3$ de dracma $=1 / 24$ de onza antigua de farmacia $=1.19$ gramos = 20 granos (Montané Marti 1998).

13. Las agallas son excrecencias producidas en las encinas por la picadura de ciertos insectos del género Gynips o Diplolepis gallae tinctoriae, L. Todas las agallas son ricas en tanino y ácido gálico; por esto se emplean para el curtido de las pieles, para fabricar tintas para tejidos y tinta de escribir y para preparación de manipulados farmacéuticos astringentes; se utilizaban como reactivos para determinar la presencia de hierro dado que colorean el agua de un tono negro ( Jourdan 1829).

14. Reactivo realizado a partir de pétalos de violetas, utilizado para medir la acidez del agua. En un medio ácido se tornaba colorada; en uno alcalino, verde. En 1750, Gabriel F. Venel, químico enciclopedista francés, "utilizando una solución patrón de ácido sulfúrico junto con un indicador, por primera vez, extracto de violetas para determinar el contenido de carbonato de las aguas" (Armijo de Castro 2012, p. 217).

15. Papel tornasol para medir acidez.

16. Carbonato potásico (Montané Marti 1998).

17. Se refiere a sedimentaciones como terrones.

18. Carbonato básico de magnesio (Montané Marti 1998).

19. Ácido sulfúrico (Montané Marti 1998). Los espíritus eran compuestos elaborados de las partes más volátiles de las sustancias.

20. Sulfato de sodio.

21. Sulfato de calcio.

22. Nitro es nitrato potásico (Montané Marti 1998). El ácido nítrico, utilizado como reactivo ya que el nitrato de mercurio en agua precipita en sulfato de mercurio.

23. El turbit mineral es sulfato mercurial (Montané Marti 1998).

24. Cloruro de plata (Montané Marti 1998).

25. El jabón se utilizaba para medir la dureza del agua. "En 1756, Francis Home (1720-1813) investigó el origen de la dureza y realizó una serie de experiencias con las sustancias que cuajaban el jabón. En su trabajo encontró hasta once agentes ablandadores como la "parte soluble de la cal", que era la más importante seguida del aceite y del "espíritu del agua de mar" (Armijo de Castro 2012, p. 218).

26. Caput mortuum es un término utilizado en alquimia en referencia a una sustancia de desecho derivada de un proceso químico, una precipitación.

27. Sulfuro ferroso (Montané Marti 1998).

28. Ver más adelante.

29. Cloro (Montané Marti 1998).

30. No se pudo hallar una referencia para este término. Podría relacionarse con la anemia. 
31. Puede observarse que la cita difiere de los análisis de Gorman en que incluye las denominaciones químicas "modernas" como hidrógeno sulfurado y ácido carbónico.

32. Nos permitimos la licencia de establecer una comparación con las investigaciones sobre los recursos hídricos actuales. Sintéticamente, los pozos del golfo y los manantiales presentan en términos generales, una hidrogeología resultante del lavado y disolución de los sedimentos de la formación Puerto Madryn (Álvarez et al. 2010, pp. 374/378): son levemente alcalinos, con presencia de bicarbonato de sodio y sulfatos -en menor cantidad al sur. Los cloruros tienen mayor presencia en la muestra de la Salina, mientras que la relación es inversamente proporcional en el caso del calcio, magnesio y sodio. En términos generales, los resultados no estandarizados del siglo XVIII presentan cierta similitud con lo analizado hoy en día. Es importante mencionar que Álvarez et al. (2010) definieron una zona de buena aptitud del agua en la que se incluye la Salina y la fuente de los manantiales en particular. Sin embargo, en esta zona se hallaron valores muy altos de potasio, arsénico y flúor que dejan estas aguas inviables para el consumo humano - de acuerdo a los estándares actuales.

33. La teoría de humores es la base de la medicina hipocrática y galénica, y entiende el estado de salud a partir del equilibrio de cuatro sustancias - humores-: bilis negra, bilis, flema y sangre, que se relacionaban con los cuatro elementos: el fuego, el aire, el agua y la tierra. Remanentes de esta teoría aún permanecían en el siglo XVIII (Andrade Lima 1996).

34. Senatore (2007) analizó. para la colonia de Floridablanca, la planificación de la salud y la alimentación en términos nutricionales; en función de las deficiencias en la alimentación, las muertes y enfermedades, concluye asimismo que las condiciones de vida eran fácilmente mejorables con una mejor alimentación según la época.

\section{ABSTRACTS}

In this work we present a documentary piece related to the Spanish colonization of the Patagonian coast at the end of the 18th Century. It is the report of the chemical analysis of waters, taken in Valdés Peninsula during the installation of San José Fort in 1779. We present the transcription of an unpublished document, followed by the lines of inquiry that come up: the method of water analysis and "translation" of the chemical elements mentioned. We contextualize the discussion in relation with the colonization of the territory and decision making about resources, the modernization of chemistry and its role in public health, specifically the importance of scorbut. This concurrence leads to the notoriety of Valdés Peninsula within the colonial scenery.

En este trabajo presentamos una pieza documental relativa a la colonización española de la costa patagónica de fines del siglo XVIII. Es el informe sobre el análisis químico de aguas tomadas en península Valdés, durante la instalación del Fuerte San José en 1779. Se presenta la trascripción del documento inédito, seguido de las líneas de indagación que surgen del mismo: el método de análisis y "traducción" de elementos químicos mencionados. Asimismo, contextualizamos la discusión en relación con la colonización del territorio, la toma de decisiones sobre los recursos, la modernización de la química y su rol en la salud pública, en particular, la importancia del escorbuto. Esta concurrencia lleva a la notoriedad de península Valdés en el escenario colonial. 
INDEX

Keywords: Valdes Peninsula, 18th Century, San José Fort, waters, scorbut

Palabras claves: Península Valdés, siglo XVIII, Fuerte San José, aguas, escorbuto 